\title{
Effect of stoichiometry on AC and DC breakdown of silicon nitride/epoxy nanocomposites
}

\author{
Fuad N. Alhabill \\ University of Chichester \\ Electronics \& Electrical Engineering, Department of Engineering and Design \\ Chichester PO19 6PE, UK \\ Alun S. Vaughan and Thomas Andritsch \\ University of Southampton \\ Tony Davies High Voltage Laboratory, Electronics and Computer Science \\ Southampton SO17 1BJ, UK \\ Nader Anani \\ University of Wolverhampton \\ School of Engineering, Telford Innovation Campus \\ Telford TF2 9NT, UK
}

\begin{abstract}
This study investigates the electrical behavior of silicon nitride/epoxy nanocomposites. It is demonstrated that the presence of the nanofiller affects the resin/hardener stoichiometry, which results in the development of different network structures throughout the matrix polymer. However, detailed analysis shows that this stoichiometric effect cannot account, alone, for the observed changes in the electrical behavior of the nanocomposite samples. A comparison between the electrical behavior of filled and the unfilled samples, where appropriate stoichiometric compensation has been applied, indicates that there is an additional effect that is exclusively a function of the nanofiller loading and which is superimposed on any matrix chemistry effects. Potential explanations for this nanoparticle effect are discussed, including: nanoparticle agglomeration; water shells around the nanoparticles; the influence of nanoparticles on matrix dynamics, structure or the free volume content of polymer interphase.
\end{abstract}

Index Terms - nanocomposites, epoxy, silicon nitride, particle interphase, conductivity, AC breakdown, DC breakdown

\section{INTRODUCTION}

WITH the continuous need for power system components characterized by higher power densities, the demands placed on insulation systems are ever-growing. Nanocomposites have received considerable research attention as a potential way to improve the dielectric performance of polymers and, thus, meet this demand for enhanced dielectric materials. Many models have been proposed to explain the electrical behavior of nanocomposites and, commonly, these have focused on the large nanoparticle/ polymer interfacial area that exists in nanocomposites and the consequent interfacial interactions that may occur between the

Manuscript received on 22 January 2021, in final form 22 January 2021, accepted xx Month 20yy. Corresponding author: F.N. Alhabill. particles and the surrounding polymer. For example, Tanaka et al [1] suggested that these interactions can result in an interaction zone or an interphase layer around the particles with modified polymeric chain dynamics and free volume content, which affect properties such as partial discharge resistance. We recently [2] suggested that a thin interphase layer within the boundaries of nanoparticles could have a critical impact on electrical behavior of nanocomposites, which has much in common with Tanaka's more recent quantum dot hypothesis [3]. Our recent work indicated that the existence of this layer beneath the nanoparticle surface, which is related to structural defects and the presence of foreign atoms and surface functional groups, can affect the local electronic density of states and, therefore, affect the charge dynamics, ultimately, throughout the whole nanocomposite system. Similar ideas concerning the introduction of localized traps by nanoparticles but within the polymer and adjacent to nanoparticle surfaces has been proposed elsewhere [4]. 
Epoxy resins are widely used as insulation materials in power devices such as cast resin transformers, switchgear, cable terminations and bushings. To produce an epoxy-based nanocomposite, nanofillers are commonly introduced into the liquid resin before the curing process. This enables effective filler/polymer mixing and thus better particle dispersion. Nanoparticles surfaces will, in general, contain moieties that do not reflect the ideal bulk chemistry of the material, such as hydroxyl and amine groups. Consequently, the inclusion of nanoparticles within an epoxy matrix may lead to chemical reactions between such particle surface groups and the active groups in the resin/hardener before and/or during the curing process. Such reactions, whilst potentially beneficially affecting interfacial bonding, will also affect the curing reaction stoichiometry by disturbing the balance between the remnant active groups in the resin and the hardener. Therefore, the incorporation of nanofillers can modify the crosslinking density and architecture of epoxy networks and, thus, affect the properties of the matrix polymer. Indeed, we have shown [5] that adding a silicon nitride nanofiller to an epoxy system results in significantly changes to the effective resin:hardener stoichiometry and have inferred that this is a consequence of the amine groups that are present on the surface of the nanoparticles reacting with a significant fraction of the resin's epoxide groups: a hardener (amine)-rich matrix system, therefore, results. Accordingly, this factor should be taken into account when analyzing the electrical behavior of thermosetting-based nanocomposites, since other studies [6] have demonstrated that changing the resin/hardener stoichiometry can affect the electrical properties of epoxy-based materials.

Water absorption is another factor that may affect the dielectric performance of epoxy nanocomposites [7, 8]. As stated above, nanofillers have a large surface area that may contain polar surface groups. Adding such particles into a polymeric matrix has been shown to increase the polar content of the material and, thus, increase the propensity for water absorption, with deleterious consequences for dielectric properties. Water can form "shells" around individual particles and, in the worst cases, form percolating networks. Such negative factors related to water absorption may interfere with or swamp any beneficial effects brought by the incorporation of nanoparticles. In particular, it has been shown experimentally how rapidly water can be absorbed by the thin specimens that are often used for experimental study [9]. The water content of such samples may, therefore, change during storage or testing time and, consequently, monitoring or understanding the effect of water absorption is essential in investigating the electrical properties of these systems.

This paper set out to examine the electrical properties of silicon nitride/epoxy nanocomposite and, specifically, to explore the consequences of the stoichiometric changes within the matrix polymer that stem from the reactive functional groups present on silicon nitride surfaces. As such, this paper extends our previous work [5], which reported on the impact of silicon nitride nanoparticles on curing reactions and consequent molecular dynamics in the same epoxy-based nanocomposite system considered here. Other factors, such as water absorption and the formation of an interphase layer are also considered.

\section{EXPERIMENTAL}

\subsection{MATERIALS}

The epoxy matrix investigated here consisted of the epoxy resin DER 332 obtained from Sigma Aldrich and Jeffamine D230 amine hardener obtained from Huntsman. The DER 332 resin is based on diglycidyl ether of bisphenol-A (DGEBA) and has an epoxide equivalent molar mass of $174 \mathrm{~g} / \mathrm{mol}$; the Jeffamine D230 hardener is a polyetheramine hardener with an amine hydrogen equivalent molar mass of $60 \mathrm{~g} / \mathrm{mol}$. The main crosslinking mechanism in this system results from the reaction of the epoxide groups in the resin with the amine groups in the hardener [6] and, therefore, based on the above epoxide and amine equivalent molar masses, the optimal stoichiometric resin:hardener ratio by weight is 1000:344.

The silicon nitride $\left(\mathrm{Si}_{3} \mathrm{~N}_{4}\right)$ nanofiller used in this study was obtained from Sigma Aldrich with a quoted particle size $<50 \mathrm{~nm}$. The surface chemistry of $\mathrm{Si}_{3} \mathrm{~N}_{4}$ is characterized by the presence of amine groups and, to a lesser extent, hydroxyl groups [2, 10]. As stated above, our preceding study [5] has demonstrated that the amine groups on the particle surfaces chemically react with epoxide groups in the resin such that a system based on a resin: hardener mass ratio of $100: 34.4$ is, effectively, rich in hardener. In order to investigate such a stoichiometric imbalance on electrical properties, formulations other than the theoretically optimum one $(100: 34.4)$ are considered here. To distinguish between different formulations, a parameter termed the hardener percentage $(H P)$ is used to indicate the percentage of hardener actually used in a sample, with respect to the theoretical stoichiometric ideal. Thus, for example, a system containing 1000 parts by weight DER 332 and 275 parts equates to a system where $H P=80 \%(275 / 344=$ 0.8 ). Table 1 summarizes the samples that were prepared for this study along with the resin:hardener ratio and filler loading used in each sample. The procedures used to prepare these samples were described in the preceding paper [5] and thus are not detailed here for brevity. The sample code used in Table 1 consists of two parts, the first part indicates the $H P$ and the other part indicates the filler loading ratio. The nanocomposite samples can be divided into two series: the first contains $2 \mathrm{wt} \%$ $\mathrm{Si}_{3} \mathrm{~N}_{4}$ at three different $H P \mathrm{~s}$; the second contains $5 \mathrm{wt} \%$ at the same HPs used in the first series. Since the addition of $\mathrm{Si}_{3} \mathrm{~N}_{4}$ nanoparticles would increase the effective amine content, the focus was on preparing nanocomposite samples with $H P<100 \%$, in an attempt to compensate for the additional amine groups present on the $\mathrm{Si}_{3} \mathrm{~N}_{4}$ particle surfaces.

Table 1. Sample details.

\begin{tabular}{ccc}
\hline Sample code & $\begin{array}{c}\text { Resin : Hardener mass } \\
\text { ratio }(\boldsymbol{H P}(\%))\end{array}$ & $\begin{array}{c}\mathbf{S i}_{3} \mathbf{N}_{\mathbf{4}} \text { filler loading } \\
(\mathbf{w t} \%)\end{array}$ \\
\hline $100 \mathrm{HP} / 0$ & $1000: 344(100 \%)$ & 0 \\
\hline $80 \mathrm{HP} / 2 \mathrm{SiN}$ & $1000: 275(80 \%)$ & 2 \\
\hline $90 \mathrm{HP} / 2 \mathrm{SiN}$ & $1000: 309(90 \%)$ & 2 \\
\hline $100 \mathrm{HP} / 2 \mathrm{SiN}$ & $1000: 344(100 \%)$ & 2 \\
\hline $80 \mathrm{HP} / 5 \mathrm{SiN}$ & $1000: 275(80 \%)$ & 5 \\
\hline $90 \mathrm{HP} / 5 \mathrm{SiN}$ & $1000: 309(90 \%)$ & 5 \\
\hline $100 \mathrm{HP} / 5 \mathrm{SiN}$ & $1000: 344(100 \%)$ & \\
\hline
\end{tabular}




\subsection{CHARACTERIZATION OF ELECTRICAL PROPERTIES}

The effect of the $\mathrm{Si}_{3} \mathrm{~N}_{4}$ nanofiller on charge transport was investigated by measuring the conductivity of each sample under a constant DC applied voltage. For this, a specimen $200 \pm 10 \mu \mathrm{m}$ in thickness was sputter coated with gold (opposing circles, $20 \mathrm{~mm}$ in diameter) on both sides, to improve the electrical contact with the measurement electrodes. The sample was then placed between opposing circular electrodes (also $20 \mathrm{~mm}$ in diameter) within the measurement apparatus, an electric field of $42 \mathrm{kV} / \mathrm{mm}$ was applied. The resulting current passing through the specimen was measured using a Keithley 6487 picoammeter over a period of $2 \mathrm{~h}$ at a sampling rate of one measurement per minute. The measurement temperature was controlled using a fan oven, but there was no control over the relative humidity during the measurement process.

DC and AC breakdown measurements were conducted by placing specimens $70 \pm 5 \mu \mathrm{m}$ in thickness between opposing $6.3 \mathrm{~mm}$ diameter steel ball bearing electrodes; the applied voltage was then increased at a constant rate until breakdown occurred. The voltage ramp rate was $100 \mathrm{~V} / \mathrm{s}$ for DC breakdown measurements and $50 \mathrm{~V} / \mathrm{s}$ for $\mathrm{AC}$ breakdown measurements. The electrodes were replaced every five measurements to avoid surface pitting from affecting the data and the test cell was immersed in silicone oil (Dow Corning 200/20CS) to prevent flashover. All the breakdown data were acquired at room temperature $\left(\sim 23^{\circ} \mathrm{C}\right)$ and a two-parameter Weibull distribution was employed statistically to analyze the results.

Before performing any of the above electrical examinations, all samples were stored under dry conditions under vacuum for at least two weeks, to remove any absorbed water.

\section{RESULTS AND DISCUSSION 3.1 DC CONDUCTIVITY}

Conductivity data obtained at $30^{\circ} \mathrm{C}$ for all samples are presented in Figure 1; equivalent measurements were also performed at room temperature $\left(\sim 23{ }^{\circ} \mathrm{C}\right)$ and $45{ }^{\circ} \mathrm{C}$. These data show that the conductivity gradually increases with time. This behavior can be ascribed to moisture absorption during the measurement process, where the samples can quickly absorb water upon being exposed to the ambient atmosphere during the measurement process [9]. Since the intention here is merely to compare in relative terms the influence of material formulation on charge transport, the obtained conductivity data were simply averaged to give a conductivity value for each system at each temperature; the resulting data are presented in Figure 2. At the three measurement temperatures, the data show the same pattern for the values of the conductivity of all samples, which confirms the validity of the obtained results. The three temperatures were chosen to be well below the glass transition temperature $\left(T_{\mathrm{g}}\right)$ of all the samples, since the emphasis here is to compare the conductivity of all the samples in their glassy state and to avoid any significant contribution through ionic conduction that is expected at temperatures near to/above $T_{\mathrm{g}}[6]$.

The data shown in Figure 2 suggest that the incorporation of $\mathrm{Si}_{3} \mathrm{~N}_{4}$ nanoparticles increases the measured conductivity and that

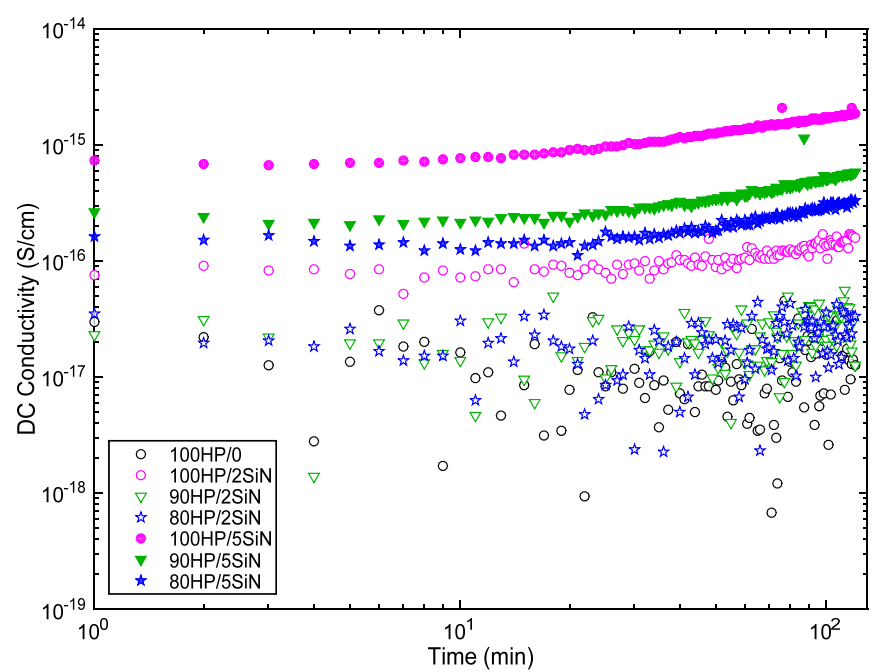

Figure 1. Conductivity measurements obtained at $30^{\circ} \mathrm{C}$ using an applied DC electric field of $42 \mathrm{kV} / \mathrm{mm}$.

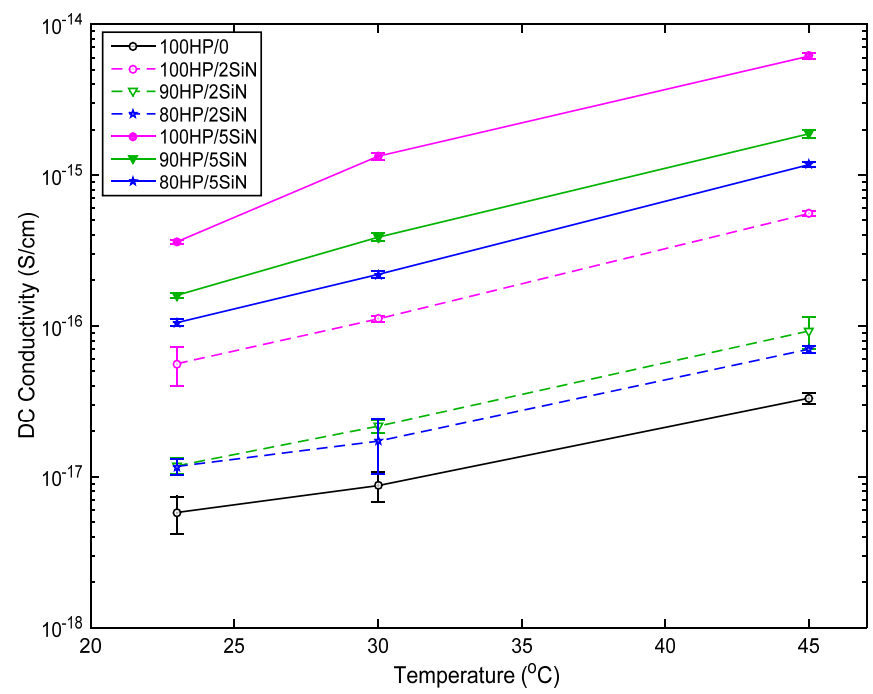

Figure 2. Average of conductivity for all the samples at different temperatures and an applied DC electric field of $42 \mathrm{kV} / \mathrm{mm}$; the error bars indicate the $95 \%$ confidence bounds of the average.

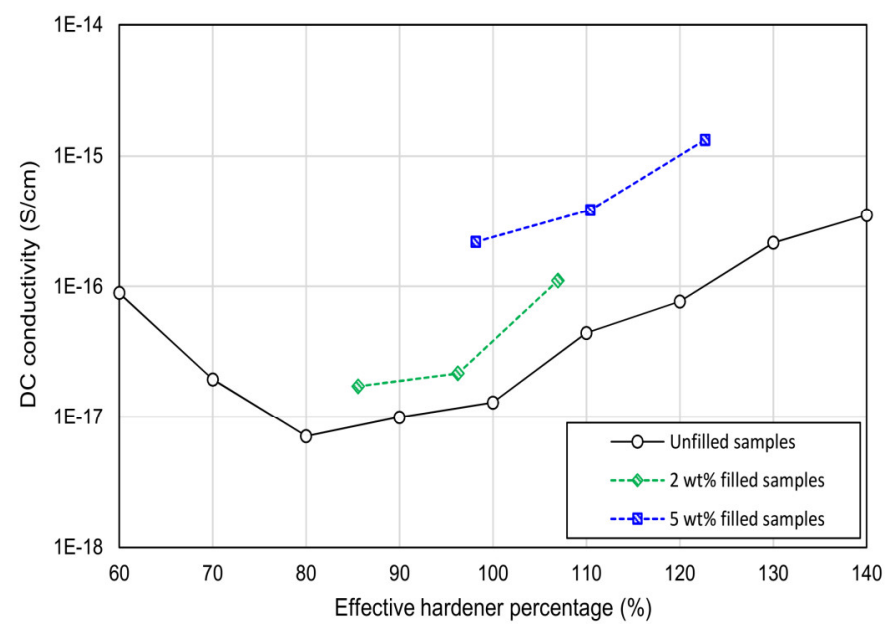

Figure 3. Influence $H P_{\text {eff. }}$ on the measured conductivity of the filled and unfilled samples: data acquired at $30^{\circ} \mathrm{C}$ and an applied DC electric field of $42 \mathrm{kV} / \mathrm{mm}$ 
the conductivity increases with increasing filler loading. Furthermore, Figures 1 and 2 show that for both the $2 \mathrm{wt} \%$ - and $5 \mathrm{wt} \%$-filled nanocomposite series, the measured conductivity decreases on reducing the $H P$ from $100 \%$ to $80 \%$. This is in line with findings reported elsewhere [6] for the same unfilled system considered here, where conductivity was found to decrease with decreasing HP. This was attributed to variations in the chemical content (amine and hydroxyl groups) of the polymer matrix that accompany changes in HP. Consequently, this behavior might signify that the increase in the DC conductivity observed in the nanocomposite samples could be related to the impact of the particles on the matrix resin : hardener stoichiometry of the matrix through reaction of amine groups on the surface of the $\mathrm{Si}_{3} \mathrm{~N}_{4}$ nanoparticles with epoxide groups from the DER 332 resin [5]. Such reactions will consume a fraction of the resin epoxide groups and thus reduce the epoxide groups available to crosslink with the hardener amine groups which, consequently, results in a polymer matrix with an effective hardener percentage $(H P$ eff $)$ that is higher than the anticipated $H P$ value shown in Table 1 . Indeed, Figures 1 and 2 show that samples $100 \mathrm{HP} / 2 \mathrm{SiN}$ and $100 \mathrm{HP} / 5 \mathrm{SiN}$ have higher DC conductivity than the reference unfilled sample $(100 \mathrm{HP} / 0)$. These two samples are expected to have higher $H P_{\text {eff }}$ than $100 \%$, i.e. higher than the $H P$ of the reference sample.

In order better to visualize the influence of the nanoparticles on conductivity, the nano-filled samples should be compared with equivalent unfilled samples, where the $H P_{\text {eff }}$ and $H P$ of the filled and unfilled samples, respectively, should be equivalent (i.e. the matrix polymer is invariant). For this, the $H P_{\text {eff }}$ of the nanocomposite samples was estimated based on calorimetric and dielectric spectroscopy results [5]. That is, the estimated $H P_{\text {eff }}$ of a nanocomposite sample is the effective hardener percentage after taking into account the $\mathrm{Si}_{3} \mathrm{~N}_{4}$ nanoparticles impact on the matrix stoichiometry. Figure 3 compares the resulting dependence of the conductivity of the nanocomposite systems on $H P_{\text {eff }}$ with equivalent data derived from unfilled samples (in unfilled samples, $\left.H P=H P_{\text {eff }}\right)$. This figure reveals that the conductivity of the filled and unfilled samples changes with respect to $H P_{\text {eff }}$ in a similar fashion. However, there is a shift to higher DC conductivity for the filled samples and this shift seems to be a function of the filler loading ratio. Consequently, this suggests that the stoichiometric impact of the $\mathrm{Si}_{3} \mathrm{~N}_{4}$ nanofiller cannot fully account for the variations seen in the DC conductivity of the nanocomposite samples. Otherwise, both the filled and unfilled samples should exhibit comparable conductivity values once the nanofiller impact on the matrix stoichiometry is accounted for, which is not the case. Therefore, Figure 3 implies that the variations seen in the DC conductivity of the filled samples is a result of a superposition of the effect of the particles on the network stoichiometry plus additional factors related directly to the presence of the particles. Such factors should be responsible for the shift to higher DC conductivity seen in the filled samples, particularly since this shift seems to be a function of the filler loading. There are several mechanisms that have been proposed in the literature to explain how nanofillers may modify electrical properties, which we will now consider in turn.

First, many researchers have suggested that nanoparticles affect the adjacent polymeric region, leading to the formation of an interphase layer with different properties to those of the unperturbed polymer matrix. For example, the multicore model [1] suggests that strong interactions between nanoparticles and polymer may restrict polymer chain dynamics around the particles and, subsequently, it has been suggested that the charge carriers may have lower mobility in this tightly bound region [11]. Indeed, Siddabattuni et al [12] have claimed that interfacial covalent bonding between the polymer and the nanoparticles leads to increasing nanocomposite resistance to charge movement and that it is this that serves to inhibit electrical breakdown; Kosmidou et al [13] proposed a correlation between the electrical resistivity and the $T_{\mathrm{g}}$ of epoxy-based nanocomposites. To conclude, the above cited studies have proposed that strong interfacial interactions (including chemical bonding), as existed in the systems considered here, would lead to improved dielectric properties, contrary to the obtained results. Furthermore, a detailed investigation [6] showed that changing the segmental dynamics of the same epoxy matrix considered here by changing the crosslink density does not correlate with the dielectric performance. In addition, the analysis of calorimetric and dielectric spectroscopy result reported in our previous paper [5] showed that such strong filler/matrix interactions do not appreciably influence the polymeric segmental dynamics, neither at a cooperative level nor at lower scale level. Therefore, we suggest that the results shown in Figures 1-3 cannot be explained by mechanisms of the sort discussed above.

Second, it has elsewhere $[13,14]$ been suggested that nanoparticles may increase the free volume present in the polymer interphase layer adjacent to nanoparticle surfaces, particularly if the nanoparticle surface chemistry is not compatible with the surrounding polymeric chains [15]. While it has been suggested that increased free volume content can degrade insulation properties [16], Nelson et al [17] have recently experimentally demonstrated that the incorporation of nanoparticles exerts only a minor influence on the free volume content of an epoxy matrix and such a marginal effect cannot account for the significant variations seen in the electrical properties of nanodielectrics. Comparable findings have also been reported elsewhere [18]. In the system considered here, the DER 332 becomes covalently bonded to $\mathrm{Si}_{3} \mathrm{~N}_{4}$ surfaces through the pendant amine groups that characterize the surface chemistry of this material, indicating high nanoparticle/matrix compatibility. Consequently, it is difficult to rationalize the observed increases in conductivity with increased local free volume and tight interfacial binding.

Third, nanoparticle agglomeration [19] is a commonly cited reason in the literature for degraded electrical properties in nanocomposites. However, as reported previously [5], the systems discussed here contain nanoparticles that are well dispersed throughout the matrix, an observation that aligns well with the favorable thermodynamic interactions that characterize the system used here. Hence, poor dispersion is not expected to be the root cause of any significant deteriorations in electrical performance.

Finally, as discussed above, water absorption [7, 8] can also result in degraded electrical performance in nanocomposites, particularly enhanced charge transport. Concerning water absorption, the surface chemistry of $\mathrm{Si}_{3} \mathrm{~N}_{4}$ nanoparticles is 


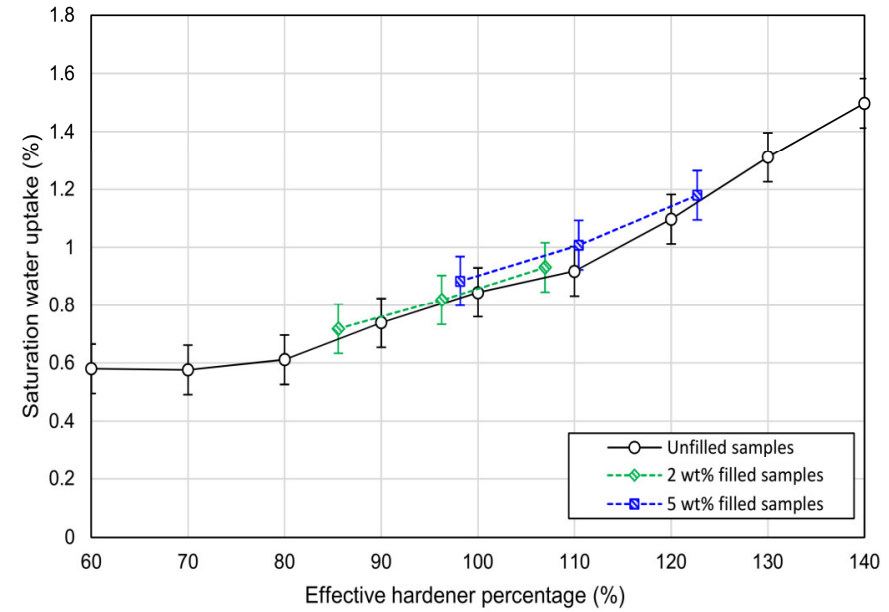

Figure 4. Saturation water uptake for the unfilled and the nanocomposite samples as a function of $H P_{\text {eff. }}$

characterized by polar groups (amine and, to a lesser extent, hydroxyl groups), which can associate strongly with any water molecules present within the system; Hosier et al [20] reported that the addition of the same nanofiller to a polyethylene matrix increased the water uptake. In the current investigation, however, care was taken to remove any absorbed water prior to any testing, by drying the prepared samples under vacuum for at least 14 days. Also, the impact of the nanoparticles on matrix curing suggests that extensive reaction of particle amine and epoxide groups from the resin occurs, with the corollary that the local chemistry will closely resemble that of the bulk. Any absorbed water should then not be strongly concentrated around the particles and, consequently, its effect on the filled and unfilled samples should be comparable. In order to test this inference, both filled and unfilled samples were exposed to ambient conditions $\left(20^{\circ} \mathrm{C}\right.$ and $\sim 60 \%$ relative humidity) and their water uptake was monitored until saturation; the results are shown in Figure 4. This shows that, when the impact of the $\mathrm{Si}_{3} \mathrm{~N}_{4}$ on matrix stoichiometry is appropriately taken into account, both the filled and unfilled samples absorb comparable amount of water. That is, the nanoparticles themselves do not exacerbate water absorption and, consequently, this is not the origin for the overall increase in the conductivity on adding $\mathrm{Si}_{3} \mathrm{~N}_{4}$ that is evident in Figures 1-3.

Our previous work has suggested that changes in the electronic surface states within nanoparticles may have a critical impact on charge transport in nanodielectrics [2]. These alterations in what we have termed the particle interphase layer are associated with the local chemistry and defect states near nanoparticle surfaces. Such changes in the localized electronic states/traps would affect charge transport close to particle surfaces and, consequently, will influence charge transport through the whole nanocomposite system, particularly if the nanoparticles are well dispersed such that inter-particle separations are small. This assertion that the structure and surface chemistry of nanoparticles can influence bulk electrical properties aligns with published experimental work [21] concerning the thermal treatment of a silica nanofiller at $1050^{\circ} \mathrm{C}$, which was reported to lead to an improvement in the dielectric performance of polyethylene-based nanocomposites. A similar

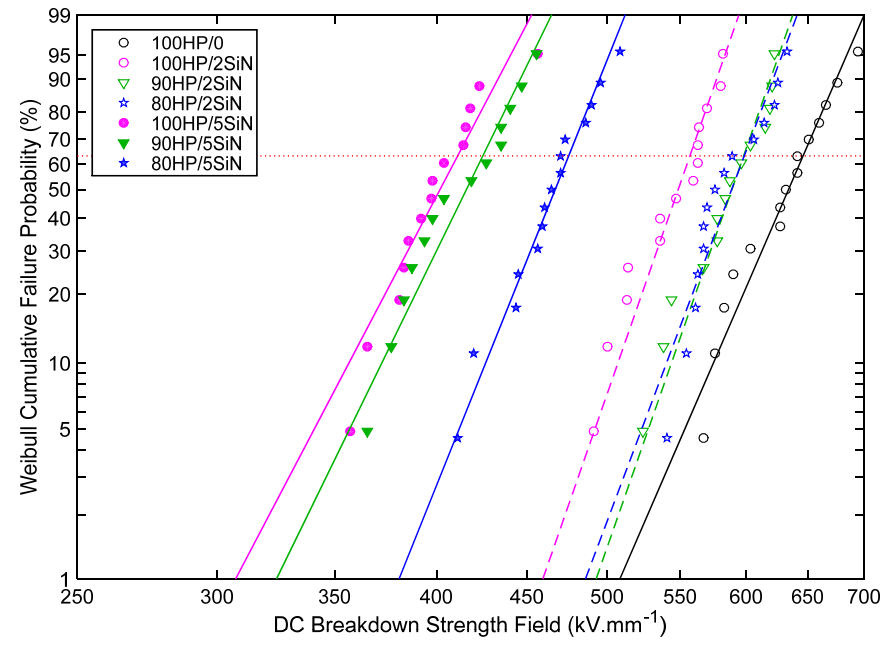

Figure 5. Weibull plot of DC breakdown measurements for all the nanocomposite samples and the reference sample.

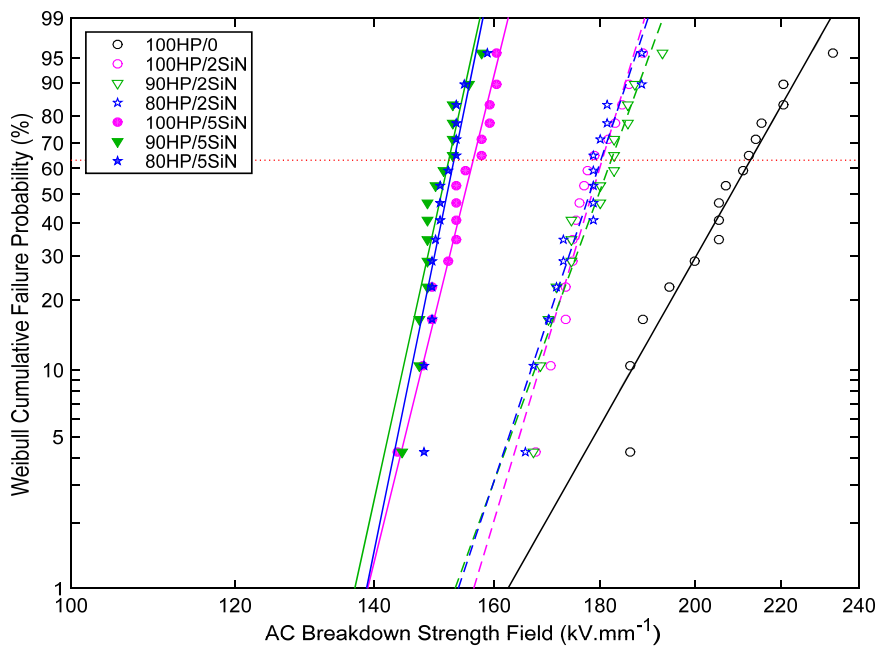

Figure 6. Weibull plot of $\mathrm{AC}$ breakdown measurements for $\mathrm{Si}_{3} \mathrm{~N}_{4}$ epoxy nanocomposites and the reference sample.

impact was found on thermally treating a silicon nitride nanofiller before adding it into an epoxy matrix [2]. Accordingly, the shift to higher conductivity values observed here in $\mathrm{Si}_{3} \mathrm{~N}_{4}$ filled epoxy systems could be related to charge transport involving additional localized states within the $\mathrm{Si}_{3} \mathrm{~N}_{4}$ nanoparticles, a concept that aligns fully with recent theoretical work by Saiz and Quirke [22].

\subsection{AND AC BREAKDOWN STRENGTH}

DC and AC breakdown data obtained from all the nanocomposite samples along with results from the unfilled reference sample are shown as Weibull plots in Figure 5 and Figure 6 , respectively. In analogy with the approach adopted in Figure 3 for sample conductivity, the variation in the resulting Weibull scale parameter with $H P_{\text {eff }}$ is shown in Figure 7 for DC breakdown and in Figure 8 for $\mathrm{AC}$ breakdown. The error bars in both figures indicate the $95 \%$ confidence bounds. These results show that the DC breakdown strength of the nanocomposite samples is affected by both the filler loading level and the matrix formulation (i.e.

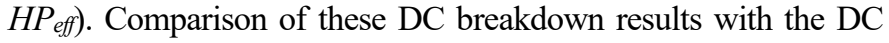




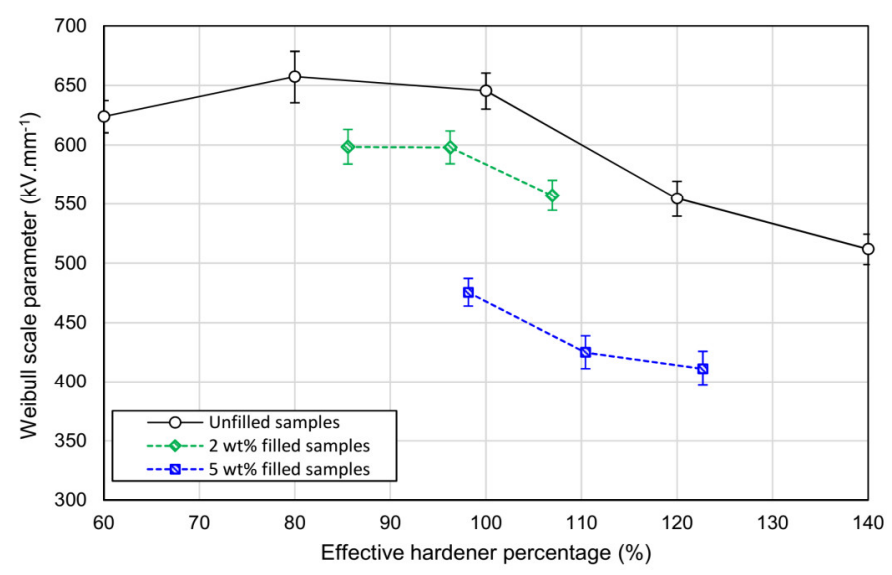

Figure 7. DC breakdown Weibull scale parameter of the filled and unfilled samples as a function of $H P_{\text {eff, }}$ the error bars indicate the $95 \%$ confidence bounds.

conductivity data presented in Figure 3 shows that systems with higher conductivity values have lower breakdown strengths and vice versa. The DC breakdown strength of the filled samples is therefore affected by both the matrix stoichiometry and the nanofiller loading level.

Conversely, the AC breakdown strength is not affected by variations in $H P_{\text {eff }}$ for both the 2 and $5 \mathrm{wt} \%$ filled nanocomposite series which is, again, in accordance with the behavior of the unfilled samples. Furthermore, the results indicate that the $\mathrm{AC}$ breakdown strength decreases with increasing filler loading. Therefore, unlike conductivity and DC breakdown strength, which are affected by both the presence of the particles and their impact on the network stoichiometry, the AC breakdown strength is only influenced by the particles themselves. To sum up, these results suggest that the DC breakdown strength of these epoxy systems is related to the conductivity, whereas the relation between the $\mathrm{AC}$ breakdown strength and the conductivity depends on the cause of the conductivity. This behavior is in line with other experimental findings reported elsewhere [23-25], which also show that $\mathrm{AC}$ breakdown strength does not correlate well with variations in DC conductivity or DC breakdown strength. For example, Grzybowski et al [23] found that water absorption caused a relatively slight reduction in the AC breakdown strength of polyethylene terephthalate, in contrast to the sharp reduction observed in DC breakdown strength. This implies that the uniform distribution of water molecules inside the insulation material has a more detrimental effect on DC breakdown than on AC breakdown. Huang [24] reported that filling a polyethylene matrix with surface-treated nano-silica degraded the AC breakdown strength, even though this reduced the DC conductivity. The authors associated this behavior with the defects and free volume that were introduced into the dielectric material by the addition of the nanoparticles. A similar conclusion was reported in [25], where it was suggested that $\mathrm{AC}$ breakdown strength is more sensitive to the presence of defects or deficiencies in insulation materials. In the systems investigated here, changing the stoichiometry, whether it is caused by changing the $H P$ directly or by the effect of adding $\mathrm{Si}_{3} \mathrm{~N}_{4}$ nanofiller, leads to a homogenous material and, thus, to a homogeneous change in the charge transport. Consequently, this is

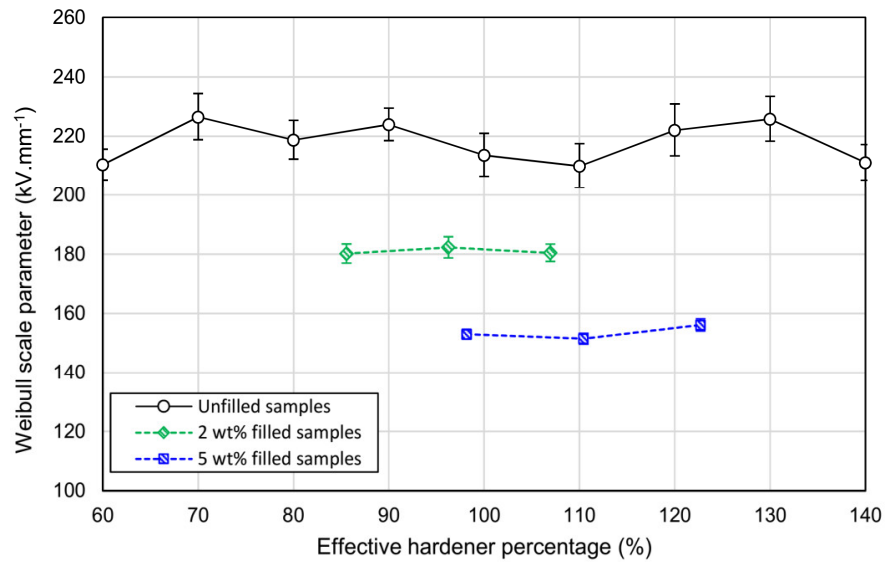

Figure 8. AC Weibull scale parameter of the filled and unfilled samples as a function of $H P$ eff, the error bars indicate the $95 \%$ confidence bounds.

not expected to produce defects in the material and, thus, does not significantly affect the $\mathrm{AC}$ breakdown strength. Conversely, the presence of the $\mathrm{Si}_{3} \mathrm{~N}_{4}$ particles is expected to increase the charge transport locally within the particle interphase, as proposed above. Such a local increase in the charge transport in a small volume within the dielectric material may form defects in this material and thus affect the $\mathrm{AC}$ breakdown strength.

\section{CONCLUSIONS}

From the work presented above, we conclude that the electrical behavior of $\mathrm{Si}_{3} \mathrm{~N}_{4}$ /epoxy nanocomposites is affected by two factors. First, the introduction of $\mathrm{Si}_{3} \mathrm{~N}_{4}$ nanoparticles perturbs the matrix resin/hardener stoichiometry, with consequences for the network architecture that forms, the retained, unreacted functional groups and, consequently, the electrical properties of the resulting system. The second factor is related directly to the presence of the nanoparticles themselves and, therefore, remains even when matrix stoichiometric effects are appropriately compensated. We suggest that this is a function of the particle loading level and the observed enhancement in charge transport may involve what we have previously termed the particle interphase region; that is a thin boundary layer within the particles that is characterized by structural defects, impurity atoms, surface functional groups, etc.

The DC and AC breakdown results reveal a good correlation between DC breakdown and conductivity, regardless of its origin, whereas the relation between the AC breakdown strength and the conductivity depends on the cause of the conductivity, i.e. whether it is due to a change in the matrix stoichiometry or due to the effect of the added nanoparticles.

Editor-in-Chief notes:

- Replace " $\mathrm{C}$ " (incorrect symbol degree $\mathrm{C}$ ) by "० $\mathrm{C}$ " in the whole manuscript!

- Don't change the text box on page 1 .

\section{REFERENCES}

[1] T. Tanaka, et al, "Proposal of a multi-core model for polymer nanocomposite dielectrics," IEEE Trans. Dielectr. Electr. Insul., vol. 12, no. 4, pp. 669-681, 2005.

[2] F. N. Alhabill, et al, "Introducing particle interphase model for describing 
the electrical behaviour of nanodielectrics," Mater. Des., vol. 158, pp. $62-$ 73, 2018.

[3] T. Tanaka, "A Quantum Dot Model for Nanoparticles in Polymer Nanocomposites," IEEE Trans. Dielectr. Electr. Insul., vol. 26, no. 1, pp. 276-283, 2019.

[4] S. Li et al, "Linking Traps to Dielectric Breakdown through Charge Dynamics for Polymer Nanocomposites," IEEE Trans. Dielectr. Electr. Insul., vol. 23, no. 5, pp. 2777-2785, 2016.

[5] F. N. Alhabill et al, "Influence of filler/matrix interactions on resin/hardener stoichiometry, molecular dynamics, and particle dispersion of silicon nitride/epoxy nanocomposites," J. Mater. Sci., vol. 53, no. 6, pp. 4144-4158, 2018.

[6] F. N. Alhabill et al, "Effect of resin/hardener stoichiometry on electrical behavior of epoxy networks," IEEE Trans. Dielectr. Electr. Insul., vol. 24, no. 6, pp. 3739-3749, 2017.

[7] C. Zou, J. C. Fothergill, and S. W. Rowe, "The effect of water absorption on the dielectric properties of epoxy nanocomposites," IEEE Trans. Dielectr. Electr. Insul., vol. 15, no. 1, pp. 106-117, 2008.

[8] L. Hui, L. S. Schadler, and J. K. Nelson, "The influence of moisture on the electrical properties of crosslinked polyethylene/silica nanocomposites," IEEE Trans. Dielectr. Electr. Insul., vol. 20, no. 2, pp. 641-653, 2013

[9] F. N. Alhabill, Dielectric Behaviour of Silicon Nitride Epoxy Nanocomposites, PhD thesis, University of Southampton, 2017.

[10] G. Busca et al, "FT-IR characterization of silicon nitride Si3N4 and silicon oxynitride SI2ON2 surfaces,” J. Mol. Struct., vol. 143, pp. 525-528, 1986.

[11] Q. Wang and G. Chen, "Effect of pre-treatment of nanofillers on the dielectric properties of epoxy nanocomposites," IEEE Trans. Dielectr. Electr. Insul., vol. 21, no. 4, pp. 1809-1816, 2014.

[12] S. Siddabattuni, T. P. Schuman, and F. Dogan, "Improved polymer nanocomposite dielectric breakdown performance through barium titanate to epoxy interface control," Mater. Sci. Eng. B, vol. 176, no. 18, pp. 14221429,2011

[13] T. V. Kosmidou et al, "Structural, mechanical and electrical characterization of epoxyamine/carbon black nanocomposites," Express Polymer Letters, vol. 2, pp. 364-372, 2008.

[14] T. P. Schuman et al, "Improved Dielectric Breakdown Strength of Covalently-Bonded Interface Polymer-Particle Nanocomposites," Compos. Interfaces, vol. 17, no. 8, pp. 719-731, 2010.

[15] S. Napolitano, E. Glynos, and N. B. Tito, "Glass transition of polymers in bulk, confined geometries, and near interfaces," Reports Prog. Phys., vol. 80, no. 3, p. 36602, 2017.

[16] J. Artbauer, "Electric strength of polymers," Journal of Physics D-Applied Physics, vol. 29, pp. 446-456, 1996.

[17] J. K. Nelson et al, "Free Volume in Nanodielectrics," IEEE 11th International Conference on the Properties and Applications of Dielectric Materials, pp. 40-43, 2015.

[18] E. do Nascimento et al, "Breakdown, free-volume and dielectric behavior of the nanodielectric coatings based on epoxy/metal oxides," Journal of Materials Science-Materials in Electronics, vol. 27, pp. 9240-9254, Sep 2016.

[19] C. Calebrese et al, "A review on the importance of nanocomposite processing to enhance electrical insulation," IEEE Trans. Dielectr. Electr. Insul., vol. 18, no. 4, pp. 938-945, 2011.

[20] I. L. Hosier et al, "The Effects of Water on the Dielectric Properties of Silicon-Based Nanocomposites," IEEE Trans. Nanotechnol., vol. 16, no. 2, pp. 169-179, 2017.

[21] M. Praeger et al, "The effects of surface hydroxyl groups in polyethylenesilica nanocomposites," IEEE Electr. Insul. Conf. (EIC), 2015, pp. 201204.

[22] F. Saiz and N. Quirke, "The excess electron in polymer nanocomposites," Phys. Chem. Chem. Phys., no.20, pp. 27528-27538, 2018.

[23] S. Grzybowski et al, "Breakdown voltage behavior of PET thermoplastics at DC and AC voltages," Proceedings IEEE Southeastcon'99. Technology on the Brink of 2000 (Cat. No.99CH36300), 1999, pp. 284-287.

[24] X. Huang, F. Liu, and P. Jiang, "Effect of nanoparticle surface treatment on morphology, electrical and water treeing behavior of LLDPE composites," IEEE Trans. Dielectr. Electr. Insul., vol. 17, no. 6, pp. $1697-$ 1704,2010

[25] B. K. Gupta, "Use of AC and DC hipot tests to assess condition of stator insulation," Proceedings:Electrical Electronics Insulation Conference and Electrical Manufacturing \& Coil Winding Conference, 1995, pp. 605-608.

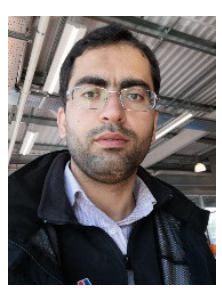

Fuad N. Alhabill received his B.Sc. degree (with distinction) in 2008 from the Islamic University of Gaza, Palestine. He worked as an electrical engineer at the Ministry of Telecommunication, Palestine, for 3 years before he was awarded Said Foundation scholarship and received the M.Sc. degree (with distinction) from the University of Southampton, UK, in 2014. In 2017 he completed his $\mathrm{PhD}$ study in high voltage power engineering at University of Southampton. He is currently working as a senior lecturer and program leader of electronics \& electrical engineering at University of Chichester, UK.

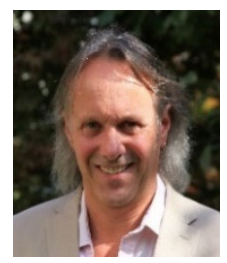

Alun Vaughan has a B.Sc. degree in chemical physics and a Ph.D. degree in polymer physics. After working at the UK's Central Electricity Research Laboratories and The University of Reading, he recently retired from the post of Professor of Dielectric Materials at the University of Southampton. He is a Fellow of the Institute of Physics, a Fellow of the IET a Senior Member of IEEE and a member of the editorial board of the IEEE DEIS Electrical Insulation Magazine. In 2016 he delivered the IEEE DEIS Eric O. Forster Memorial Lecture.

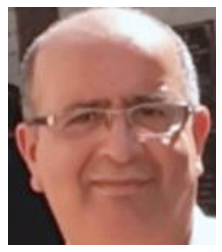

Nader Anani received his B.Sc. (Hons) degree in electrical and electronic engineering from Newcastle University (UK) in 1984 and the MSc degree in Microelectronics from the University of Manchester (UK), in October 1987 and his PhD in 2013. He took up various lecturing and research posts at UK and overseas universities and is currently an associate professor with the University of Wolverhampton, UK. His research interests are in renewable energy and power electronics. He has authored and co-authored more than 80 articles and he is a senior member of the IEEE.

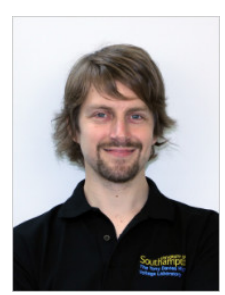

Thomas Andritsch (M'11) received the Dipl.-Ing. Degree in Electrical Engineering from Graz University of Technology in 2006 and his Ph.D. degree in the same field from Delft University of Technology in 2010. He is currently working as an Associate Professor at the University of Southampton with focus on advanced materials for high voltage applications and plant. His research interests cover polymer-based electrical insulation materials, including nanodielectrics, electroactive polymers and syntactic foams, as well as liquid insulation systems and nanofluids. Thomas is currently the chair of the UK \& Ireland IEEE DEIS Chapter, elected member to the IEEE DEIS AdCom and UK representative to CIGRE WGs D1.73 \& B1/D1.75. 